\title{
Cavity-enhanced detection of transient absorption signals
}

\author{
Fernanda C. Rodrigues-Machado ${ }^{1 *}$, Pauline Pestre ${ }^{1}$, Liam Scanlon $^{1}$, Shirin A. Enger ${ }^{2}$, Jack C. Sankey ${ }^{1}$, and Lilian I. \\ Childress $^{1 * *}$ \\ ${ }^{1}$ Department of Physics, McGill University, Montreal, Canada \\ ${ }^{2}$ Medical Physics Unit, McGill University, Montreal, Canada
}

\begin{abstract}
We present a simple, high-duty-cycle, cavity-enhanced optical absorption measurement technique based on delay-limited Pound-Drever-Hall (PDH) sideband locking. The chosen circuit naturally provides realtime readout of the amplitude quadrature of the PDH error signal, which can be mapped onto the cavity's internal loss rate while using the phase quadrature to lock sideband frequency to the cavity mode. Our proofof-concept device comprises a 5-cm-long Fabry-Perot cavity with a $450 \mathrm{kHz}$ bandwidth (finesse 6800, 350 ns power ringdown), and a feedback bandwidth of several $\mathrm{MHz}$, limited primarily by the group delay of our electronics. This technique could readily be applied to other optical resonators such as fiber cavities, with potential applications in radiation dosimetry.
\end{abstract}

\section{Introduction}

We work on the development of a time resolved cavityenhanced absorption sensing technique based on PoundDrever-Hall (PDH) sideband locking.

PDH locking [1] relies on phase modulation of a laser beam and posterior demodulation of the power reflected from an optical cavity by mixing with a local oscillator at the same modulation frequency. With the correct choice of phase for the local oscillator (corresponding to the phase quadrature of the power), the resulting mixed-down voltage provides a derivative-like signal that can be used to lock the cavity on resonance to an incoming laser beam. Here, we lock the cavity to one of the sidebands generated by phase modulation, which allows a high locking bandwidth $(\sim \mathrm{MHz})[2]$.

In addition to creating the locking error signal (phase quadrature), we use a $\pi / 2$-shifted signal and a second mixer to get the PDH amplitude quadrature. The amplitude quadrature has a Lorentzian shape about the detuning of the sideband (at modulation frequency $\Omega$ ), and provides a non-zero signal when the sideband is locked to the cavity. This signal carries real-time information from the cavity and can be mapped onto the cavity's internal losses.

\section{Experimental apparatus}

We use a 5-cm-long, single-sided Fabry-Perot cavity with finesse 6800 to demonstrate the technique (Fig. 1.a). A $1550 \mathrm{~nm}$ laser beam is phase-modulated by an electrooptical modulator (EOM), which receives an RF signal $(\Omega \sim 1 \mathrm{GHz})$ from a voltage-controlled oscillator (VCO). The laser beam reaches the cavity and the reflected light

\footnotetext{
*e-mail: fernanda.rodriguesmachado@mcgill.ca

**e-mail: lilian.childress@mcgill.ca
}

is collected by a fast photodiode. Half the signal coming from the photodiode is used for PDH feedback $\left(\mathrm{V}_{\mathrm{y}}\right)[1,2]$, while the other half is phase-shifted by $\pi / 2$ and mixed with the VCO signal to generate the amplitude quadrature $\left(\mathrm{V}_{\mathrm{x}}\right)$. When the cavity is locked to one of the sidebands, the $V_{x}$ signal slowly fluctuates about a DC offset, and varies linearly with cavity lifetime.

With the high bandwidth provided by sideband PDH locking, we can keep the cavity locked on resonance for several hours, allowing the detection of transient signals.

\section{Results}

We measure the sensitivity noise floor with power spectral densities and translate the amplitude quadrature voltage signal to absorption (units of $\mathrm{cm}^{-1}$ ) by calibrating the apparatus with swept ring-down measurements [3].

The expected shot-noise limit in units of $\mathrm{cm}^{-1} / \mathrm{Hz}^{1 / 2}$ is calculated by

$$
\left\langle S_{\alpha}\right\rangle=a \sqrt{2 e \eta P_{P D} Z^{2} \xi}
$$

where $a$ is a conversion factor determined by calibration (units of $\mathrm{cm}^{-1} / \mathrm{V}$ ), $e$ is the electron charge, $\eta$ is the photodiode responsivity (in A/W), $P_{P D}$ is the total power reaching the photodiode, $Z$ is the diode transimpedance (in V/A) and $\xi$ accounts for the unitless circuit components power gains.

Figure 1.b shows our sensitivity floor. The locked (blue) curve is the signal we use to monitor cavity absorption. Our measurements are currently limited by laser classical noise, achieving sensitivities on the order of $10^{-10} \mathrm{~cm}^{-1} / \mathrm{Hz}^{1 / 2}$, within a factor of $<30$ from shot-noise limit (red curve, confirmed by results from equation 1 green curve). These results are comparable to the most sensitive absorption measurements reported to date $[4,5]$. 
a)

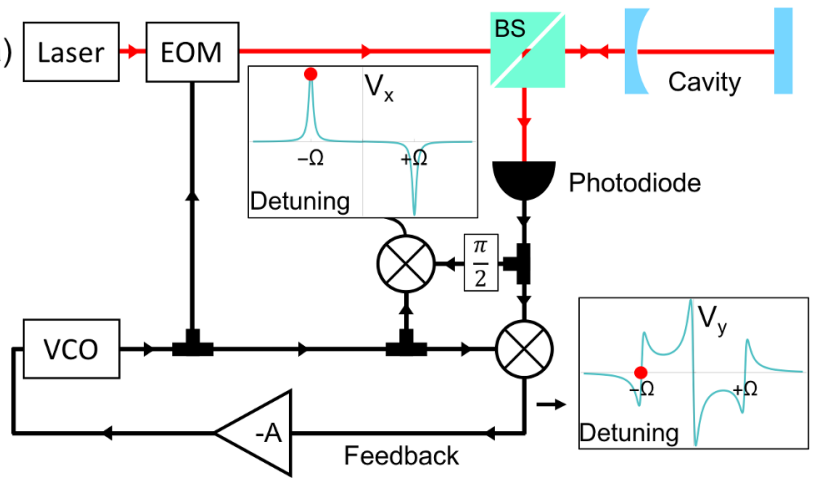

b)

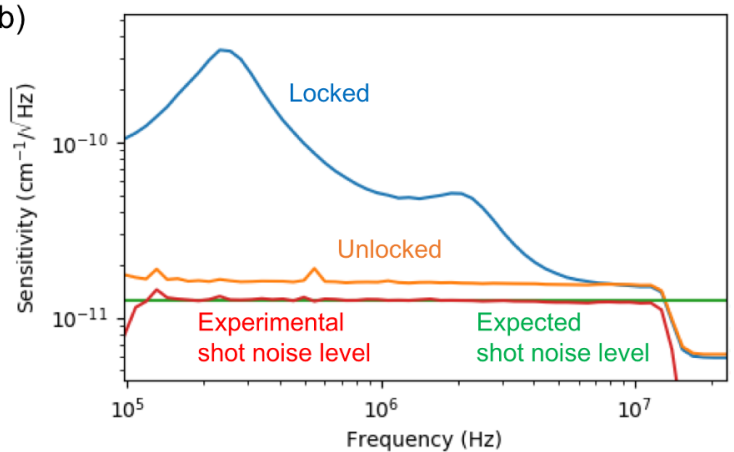

Figure 1. a) Experimental setup (simplified) for sideband PDH locking and intra-cavity absorption sensing. EOM: electro-optical modulator. BS: non-polarizing beamsplitter. VCO: voltage-controlled oscillator. -A: feedback controller. $\pi / 2$ indicates a phase shift. Insets show PDH amplitude quadrature $\left(\mathrm{V}_{\mathrm{x}}\right)$ and phase quadrature $\left(\mathrm{V}_{\mathrm{y}}\right)$. Red dots indicate the lock point on the lower sideband. b) Setup noise power spectral densities converted to units of absorption. Blue: noise floor of the system while the laser is locked to the cavity. Orange: unlocked laser noise (quantum limited) and electronics noise. Red: laser shot noise level (electronics subtracted). Green: shot noise limit calculated with equation 1.

\section{Outlook}

We are currently looking into phase-detection techniques (e.g. with amplitude modulation of the laser beam) to acquire direct absorption, calibration-free measurements. We are also exploring noise cancellation techniques to subtract the classical noise seen in our locked measurements (Fig. 1.b - blue) and achieve shot-noise-limited measurements, even at lower frequencies.

Our described technique can be applied to other optical resonators such as fiber cavities, which would provide micrometer spatial resolution and $\mathrm{GHz}$ bandwidth. In the near future, we aim to employ such fiber cavities in studies of radiation dosimetry. The continuous monitoring of the cavity potentially allows the detection of solvated electrons, short lived entities generated in water when radiation is incident. This highly sensitive technique would allow the real-time detection of low radiation doses ( 1mGy) against an absorptive background.

\section{References}

[1] E.D. Black, American journal of physics 69, 79 (2001)

[2] C. Reinhardt, T. Müller, J.C. Sankey, Optics express 25, 1582 (2017)

[3] Y. He, B. Orr, Applied Physics B 75, 267 (2002)

[4] J. Ye, L.S. Ma, J.L. Hall, JOSA B 15, 6 (1998)

[5] L.S. Ma, J. Ye, P. Dubé, J.L. Hall, JOSA B 16, 2255 (1999) 